\title{
Normal Families of Meromorphic Functions Concerning Higher Derivative and Shared Values
}

\author{
Wei Chen, ${ }^{1}$ Wenjun Yuan, ${ }^{2}$ and Honggen Tian' \\ ${ }^{1}$ School of Mathematics Sciences, Xinjiang Normal University, Urumqi, Xinjiang 830054, China \\ ${ }^{2}$ School of Mathematics and Information Sciences, Guangzhou University, Guangzhou, Guangdong 510006, China
}

Correspondence should be addressed to Honggen Tian; tianhg@xjnu.edu.cn

Received 3 July 2013; Revised 12 September 2013; Accepted 12 September 2013

Academic Editor: Alberto Fiorenza

Copyright (C) 2013 Wei Chen et al. This is an open access article distributed under the Creative Commons Attribution License, which permits unrestricted use, distribution, and reproduction in any medium, provided the original work is properly cited.

We study the normal families related to a Hayman conjecture of higher derivative and concerning shared values and get two normal criteria. Our results improve the related theorems which were obtained independently, respectively by Fang and Yuan (2001), Yuan et al. ((2011) and (2012)), Wang et al. (2011), and Qiu et al. (2012). Meanwhile, some examples are given to show the sharpness of our results.

\section{Introduction and Main Results}

Let $f(z)$ and $g(z)$ be two nonconstant meromorphic functions in a domain $D \subseteq \mathbb{C}$, and let $a$ be a finite complex value. We say that $f$ and $g$ share $a \mathrm{CM}$ (or IM) in $D$ provided that $f-a$ and $g-a$ have the same zeros counting (or ignoring) multiplicity in $D$. When $a=\infty$, the zeros of $f-a$ mean the poles of $f$ (see [1]). It is assumed that the reader is familiar with the standard notations and the basic results of Nevanlinna's value-distribution theory ([1] or [2-4]).

It is very interesting to find normality criteria from the point of view of shared values. In this area, Schwick [5] first proved an interesting result that a family of meromorphic functions in a domain is normal in which every function shares three distinct finite complex numbers with their first derivative. And later, more results about normality criteria concerning shared values have emerged; for instance, see [68]. In recent years, this subject has attracted the attention of many researchers worldwide.

We now first introduce a normality criterion related to a Hayman normal conjecture [9].

Theorem 1. Let $\mathscr{F}$ be a family of holomorphic (meromorphic) functions defined in a domain, $n \in \mathbb{N}, a \neq 0$, and $b \in \mathbb{C}$. If $f^{\prime}(z)+a f^{n}(z)-b$ does not vanish in $D$ for each function $f(z) \in$ $\mathscr{F}$ and $n \geq 2(n \geq 3)$, then $\mathscr{F}$ is normal in $D$.
The results for the holomorphic case are due to Drasin [10] for $n \geq 3$, Pang [11] for $n=3$, Chen and Fang [12] for $n=$ 2, Ye [13] for $n=2$, and Chen and Gu [14] for the generalized result with $a$ and $b$ replaced by meromorphic functions. The results for the meromorphic case are due to $\mathrm{Li}$ [15], Li [16], and Langley [17] for $n \geq 5$, Pang [11] for $n=4$, Chen and Fang [12] for $n=3$, and Zalcman [18] for $n=3$, obtained independently.

When $n=2$ and $\mathscr{F}$ is meromorphic, Theorem 1 is not valid in general. Fang and Yuan [19] gave an example to show this and got a special result below.

Example 2. The family of meromorphic functions $\mathscr{F}=$ $\left\{f_{j}(z)=j z /(\sqrt{j} z-1)^{2}: j=1,2, \ldots,\right\}$ is not normal in $D=\{z:|z|<1\}$. This is deduced by $f_{j}^{\#}(0)=j \rightarrow \infty$, as $j \rightarrow \infty$ and Marty's criterion [2], although for any $f_{j}(z) \in$ $\mathscr{F}, f_{j}^{\prime}+f_{j}^{2}=j(\sqrt{j} z-1)^{-4} \neq 0$.

Here $f^{\#}(\xi)$ denotes the spherical derivative

$$
f^{\#}(\xi)=\frac{\left|f^{\prime}(\xi)\right|}{1+|f(\xi)|^{2}} .
$$

Theorem 3. Let $\mathscr{F}$ be a family of meromorphic functions in a domain $D$, and $a \neq 0, b \in \mathbb{C}$. If $f^{\prime}(z)+a f^{2}(z)-b$ does not 
vanish in $D$ and the poles of $f(z)$ are of multiplicity $\geq 3$ for each $f(z) \in \mathscr{F}$, then $\mathscr{F}$ is normal in $D$.

In 2008, by the ideas of shared values, Zhang [8] proved the following.

Theorem 4. Let $\mathscr{F}$ be a family of meromorphic (holomorphic) functions in $D$, let $n$ be a positive integer and let $a, b$ be two finite complex numbers such that $a \neq 0$. If $n \geq 4(n \geq 2)$ and for every pair of functions $f$ and $g$ in $\mathscr{F}, f^{\prime}-a f^{n}$ and $g^{\prime}-a g^{n}$ share the value $b I M$, then $\mathscr{F}$ is normal in $D$.

Example 5 (see [8]). The family of meromorphic functions $\mathscr{F}=\left\{f_{j}(z)=1 / \sqrt{j}(z-1 / j): j=1,2, \ldots,\right\}$ is not normal in $D=\{z:|z|<1\}$. Obviously $f_{j}^{\prime}-f_{j}^{3}=-z / \sqrt{j}(z-1 / j)^{3}$. So for each pair of $m, j, f_{j}^{\prime}-f_{j}^{3}$ and $f_{m}^{\prime}-f_{m}^{3}$ share the value 0 in $D$, but $\mathscr{F}$ is not normal at the point $z=0$, since $f_{j}^{\#}(0)=$ $2(\sqrt{j})^{3} /(1+j) \rightarrow \infty$, as $j \rightarrow \infty$.

Remark 6. Example 5 shows that Theorem 4 is not valid when $n=3$, and the condition $n=4$ is best possible for meromorphic case.

In 2011, Yuan et al. [20] and Wang et al. [21] proved the following theorems, independently, respectively.

Theorem 7 (see [20,21]). Let $\mathscr{F}$ be a family of meromorphic functions in $D$ and $a, b$ two finite complex numbers such that $a \neq 0$. Suppose that each $f \in \mathscr{F}$ has no simple pole. If $f^{\prime}-a f^{3}$ and $g^{\prime}-a g^{3}$ share the value $b$ IM for every pair of functions $f$ and $g$ in $\mathscr{F}$, then $\mathscr{F}$ is normal in $D$.

Theorem 8 (see [20]). Let $\mathscr{F}$ be a family of meromorphic functions in $D$ and $a$ and $b$ two finite complex numbers such that $a \neq 0$. Suppose that each $f \in \mathscr{F}$ admits zeros of multiple and the poles of multiplicity at least 3. If $f^{\prime}-a f^{2}$ and $g^{\prime}-a g^{2}$ share the value $b I M$ for every pair of functions $f$ and $g$ in $\mathscr{F}$, then $\mathscr{F}$ is normal in $D$.

Lately, Yuan et al. [22] and Qiu et al. [23] studied this result, independently, respectively, in which the derivative $f^{\prime}$ was replaced by $k$ th derivative $f^{(k)}$, and they got the following results.

Theorem 9 (see [22]). Let $\mathscr{F}$ be a family of meromorphic functions in $D$, and let $k(\geq 2)$ and $n(\geq k+2)$ be two positive integers. Let $a(\neq 0)$ and $b$ be two finite complex numbers. If

(i) $f^{(k)}-a f^{n}$ and $g^{(k)}-a g^{n}$ share $b$ IM in $D$ for every pair of functions $f$ and $g$ in $\mathscr{F}$,

(ii) $f$ has no simple pole and no zero of multiplicity less than $k$ in $D$ for every function $f \in \mathscr{F}$, then $\mathscr{F}$ is normal in $D$.

Theorem 10 (see $[22,23])$. Let $\mathscr{F}$ be a family of meromorphic functions in $D, k(\geq 2)$ and $n(\geq k+3)$ be two positive integers. Let $a(\neq 0)$ and $b$ be two finite complex numbers. If

(i) $f^{(k)}-a f^{n}$ and $g^{(k)}-a g^{n}$ share $b$ IM in D for every pair of functions $f$ and $g$ in $\mathscr{F}$, (ii) $f$ has no zero of multiplicity less than $k$ in $D$ for every function $f \in \mathscr{F}$, then $\mathscr{F}$ is normal in $D$.

It is natural to ask whether the condition $n \geq k+2$ or $n \geq k+3$ in the previous theorems can be reduced. In this paper, we study this problem and get the following results.

Theorem 11 (main theorem). Let $D$ be a domain in $\mathbb{C}$ and let $\mathscr{F}$ be a family of meromorphic functions in $D$. Let $k, n, d \in$ $\mathbb{N}^{+}, n \geq 3, d \geq(k+1) /(n-2)$ and let $a, b$ be two finite complex numbers with $a \neq 0$. Suppose that every $f \in \mathscr{F}$ has all its zeros of multiplicity at least $k$ and all its poles of multiplicity at least d. If $f^{(k)}-a f^{n}$ and $g^{(k)}-a g^{n}$ share the value $b$ IM for every pair of functions $(f, g)$ of $\mathscr{F}$, then $\mathscr{F}$ is a normal family in $D$.

Remark 12. When $n \geq k+2$ or $n \geq k+3$, we have $d=1$ or 2 , respectively. It follows that Theorem 11 generalizes Theorems $4,7,9$, and 10 .

Theorem 13 (main theorem). Let $D$ be a domain in $\mathbb{C}$ and let $\mathscr{F}$ be a family of meromorphic functions in D. Let $k \in \mathbb{N}^{+}$ and $a, b$ be two finite complex numbers with $a \neq 0$. Suppose that every $f \in \mathscr{F}$ has all its zeros of multiplicity at least $k+1$ and all its poles of multiplicity at least $k+2$. If $f^{(k)}-a f^{2}$ and $g^{(k)}-a g^{2}$ share the valueb IM for every pair offunctions $(f, g)$ of $\mathscr{F}$, then $\mathscr{F}$ is a normal family in $D$.

Remark 14. When $k=1$, it follows that Theorem 13 generalizes Theorem 8 .

Example 15 (see [23]). Let $n, k \geq 2$ be two positive integers and $a$ a nonzero complex constant. The family of meromorphic functions is $\mathscr{F}=\left\{f_{j}(z)=j z^{k-1}: j=1,2, \ldots,\right\}$, $D=\{z:|z|<1\}$. Obviously, for each pair of $m, j, f_{j}^{(k)}-a f_{j}^{n}$ and $f_{m}^{(k)}-a f_{m}^{n}$ share the value 0 in $D$, but $\mathscr{F}$ is not normal.

Example 16. Let $k$ be a positive integer and $a, b$ two nonzero complex constants such that $b_{j}=a /(-1)^{k}((2 k) ! / k !) j^{k}$. The family of meromorphic functions is $\mathscr{F}=\left\{f_{j}(z)=1 /(j z-\right.$ $\left.\left.b_{j}\right)^{k+1}: j=1,2, \ldots\right\}, D=\{z:|z|<1\}$. Obviously, for each pair of $m, j, f_{j}^{(k)}-a f_{j}^{2}$ and $f_{m}^{(k)}-a f_{m}^{2}$ share the value 0 in $D$, but $\mathscr{F}$ is normal since $f^{\#}(0) \rightarrow \infty$ as $j \rightarrow \infty$.

Remark 17. Example 15 shows that the condition that $f(z)$ admits zeros of multiplicity at least $k$ is best in Theorem 11 . For the case $k=1, n=3$, Example 5 shows that the condition that $f(z)$ admits poles of multiplicity at least $d$ is sharp in Theorem 11. For the case $n=2$, Example 16 shows that the condition that $f(z)$ admits poles of multiplicity at least $k+2$ is sharp in Theorem 13. For the case $k=1, n=2$, Example 2 shows that the condition that $f(z)$ admits zeros of multiplicity at least $k+1$ in Theorem 13 is sharp.

\section{Preliminary Lemmas}

In order to prove our results, we need the following lemmas. The first is the extended version Zalcman's [24] concerning normal families. 
Lemma 1 (see [25]). Let $\mathscr{F}$ be a family of meromorphic functions on the unit disc satisfying all zeros of functions in $\mathscr{F}$ having multiplicity $\geq p$ and all poles of functions in $\mathscr{F}$ having multiplicity $\geq q$. Let $\alpha$ be a real number satisfying $-q<\alpha<p$. Then $\mathscr{F}$ is not normal at 0 if and only if there exist

(a) a number $0<r<1$;

(b) points $z_{n}$ with $\left|z_{n}\right|<r$;

(c) functions $f_{n} \in \mathscr{F}$;

(d) positive numbers $\rho_{n} \rightarrow 0$

such that $g_{n}(\zeta):=\rho_{n}^{-\alpha} f_{n}\left(z_{n}+\rho_{n} \zeta\right)$ converges spherically uniformly on each compact subset of $\mathbb{C}$ to a nonconstant meromorphic function $g(\zeta)$, whose all zeros have multiplicity $\geq p$ and all poles have multiplicity $\geq q$ and order is at most 2 .

Lemma 2. Let $f(z)$ be a meromorphic function such that $f^{(k)}(z) \not \equiv 0$ and $c \in \mathbb{C} \backslash\{0\}, k, n, d \in \mathbb{N}^{+}$with $n \geq 3$, $d \geq(k+1) /(n-2)$. If all zeros of $f$ are of multiplicity at least $k$ and all poles of $f$ are of multiplicity at least $d$, then

$$
T(r, f) \leq \frac{1}{k} N\left(r, \frac{1}{f}\right)+\bar{N}\left(r, \frac{1}{f^{(k)}-c f^{n}}\right)+S(r, f),
$$

where $S(r, f)=o(T(r, f))$, as $r \rightarrow \infty$, possibly outside a set with finite linear measure.

Proof. Set

$$
\Phi(z):=\frac{f^{(k)}(z)}{c f^{n}(z)} .
$$

Since $f^{(k)}(z) \not \equiv 0$, we have $\Phi(z) \not \equiv 0$. Thus,

$$
f^{n}(z)=\frac{f^{(k)}(z)}{c \Phi(z)}
$$

Hence,

$$
\begin{aligned}
n m(r, f) & =m\left(r, f^{n}\right) \leq m\left(r, \frac{f^{(k)}}{\Phi}\right)+\log ^{+} \frac{1}{|c|} \\
& \leq m\left(r, \frac{1}{\Phi}\right)+m\left(r, f^{(k)}\right)+\log ^{+} \frac{1}{|c|} \\
& \leq m\left(r, \frac{1}{\Phi}\right)+m\left(r, \frac{f^{(k)}}{f}\right)+m(r, f)+\log ^{+} \frac{1}{|c|} .
\end{aligned}
$$

So that

$$
(n-1) m(r, f) \leq m\left(r, \frac{1}{\Phi}\right)+m\left(r, \frac{f^{(k)}}{f}\right)+\log ^{+} \frac{1}{|c|} .
$$

On the other hand, (4) gives

$$
\begin{aligned}
n N(r, f) & \leq N\left(r, f^{n}\right)=N\left(r, \frac{f^{(k)}}{\Phi}\right) \\
& \leq N\left(r, f^{(k)}\right)+N\left(r, \frac{1}{\Phi}\right)-\bar{N}\left(r, \Phi=f^{(k)}=0\right)
\end{aligned}
$$

where $\bar{N}\left(r, \Phi=f^{(k)}=0\right)$ denotes the counting function of zeros of both $\Phi$ and $f^{(k)}$.

We obtain

$$
\begin{aligned}
n N(r, f) \leq & N(r, f)+k \bar{N}(r, f) \\
& +N\left(r, \frac{1}{\Phi}\right)-\bar{N}\left(r, \Phi=f^{(k)}=0\right), \\
(n-1) N(r, f) \leq & k \bar{N}(r, f) \\
& +N\left(r, \frac{1}{\Phi}\right)-\bar{N}\left(r, \Phi=f^{(k)}=0\right) .
\end{aligned}
$$

By (4), we have

$$
\begin{aligned}
& \bar{N}(r, \varphi)+\bar{N}\left(r, \frac{1}{\varphi}\right) \\
& \quad \leq \bar{N}\left(r, \frac{1}{f}\right)+\bar{N}(r, f)+\bar{N}\left(r, \Phi=f^{(k)}=0\right) .
\end{aligned}
$$

From (6) (9), we obtain

$$
\begin{aligned}
(n-1) & T(r, f) \\
\leq & k \bar{N}(r, f)+T\left(r, \frac{1}{\Phi}\right)-\bar{N}\left(r, \Phi=f^{(k)}=0\right)+S(r, f) \\
\leq & k \bar{N}(r, f)+T(r, \Phi)-\bar{N}\left(r, \Phi=f^{(k)}=0\right)+S(r, f) \\
\leq & k \bar{N}(r, f)+\bar{N}\left(r, \frac{1}{\Phi}\right)+\bar{N}(r, \Phi)+\bar{N}\left(r, \frac{1}{\Phi-1}\right) \\
& -\bar{N}\left(r, \Phi=f^{(k)}=0\right)+S(r, f) \\
\leq & (k+1) \bar{N}(r, f)+\bar{N}\left(r, \frac{1}{f}\right) \\
& +\bar{N}\left(r, \frac{1}{f^{(k)}-c f^{n}}\right)+S(r, f) .
\end{aligned}
$$

Since all zeros and poles of $f$ are multiplicities at least $k$ and $d$, we get

$$
\begin{aligned}
\bar{N}(r, f) \leq \frac{1}{d} N(r, f) & \leq \frac{1}{d} T(r, f) \leq \frac{n-2}{k+1} T(r, f), \\
\bar{N}\left(r, \frac{1}{f}\right) & \leq \frac{1}{k} N\left(r, \frac{1}{f}\right) .
\end{aligned}
$$

So that

$$
T(r, f) \leq \frac{1}{k} N\left(r, \frac{1}{f}\right)+\bar{N}\left(r, \frac{1}{f^{(k)}-c f^{n}}\right)+S(r, f) .
$$

Lemma 3. Let $f(z)$ be a transcendental meromorphic function such that $f^{(k)}(z) \neq 0$. Let $k \in \mathbb{N}^{+}$and $c \in \mathbb{C} \backslash\{0\}$. If all zeros of $f$ are of multiplicity at least $k+1$ and all poles of $f$ are of multiplicity at least $k+2$, then $f^{(k)}-c f^{2}$ has infinitely many zeros. 
Proof. Suppose that $f^{(k)}-c f^{2}$ has only finitely many zeros; then $N\left(r, 1 /\left(f^{(k)}-c f^{2}\right)\right)=S(r, f)$. Clearly, an arbitrary zero of $f$ is a zero of $f^{(k)}-c f^{2}$ since all zeros of $f$ are of multiplicity at least $k+1$; then we can deduce that $f$ has only finite zeros, so $N(r, 1 / f)=O(\log r)=S(r, f)$.

Set

$$
\Phi(z):=\frac{f^{(k)}(z)}{c f^{2}(z)} .
$$

Similarly, with the proof of Lemma 2, we can get

$$
\begin{aligned}
T(r, f) \leq & (k+1) \bar{N}(r, f)+\bar{N}\left(r, \frac{1}{f}\right) \\
& +\bar{N}\left(r, \frac{1}{f^{(k)}-c f^{2}}\right)+S(r, f) .
\end{aligned}
$$

Since all poles of $f$ are multiplicities at least $k+2$, we obtain

$$
\bar{N}(r, f) \leq \frac{1}{k+2} N(r, f) \leq \frac{1}{k+2} T(r, f),
$$

so that

$$
\begin{aligned}
T(r, f) \leq & (k+2) \bar{N}\left(r, \frac{1}{f}\right) \\
& +(k+2) \bar{N}\left(r, \frac{1}{f^{(k)}-c f^{2}}\right)+S(r, f)=S(r, f) .
\end{aligned}
$$

This is contradicting with the fact that $f$ is transcendental.

Hence, Lemma 3 is proved completely.

Lemma 4. Let $f(z)$ be a nonconstant rational function such that $f^{(k)}(z) \neq \equiv$. Let $a \in \mathbb{C} \backslash\{0\}$, and $k, n, m \in \mathbb{N}^{+}$with $n \geq 2$ and $m \geq(k+2) /(n-1)$. If $f \neq 0$ and all poles of $f$ are of multiplicity at least $m$, then $f^{(k)}-a f^{n}$ has at least two zeros.

Proof. Suppose, to the contrary, that $f^{(k)}-a f^{n}$ has at most one zero.

Since $f \neq 0$, we get that $f$ is a rational but not a polynomial.

Case 1. If $f^{(k)}-a f^{n}$ has only zero $z_{0}$ with multiplicity $l$, set

$$
f(z)=\frac{A}{\left(z-z_{1}\right)^{\beta_{1}}\left(z-z_{2}\right)^{\beta_{2}} \cdots\left(z-z_{t}\right)^{\beta_{t}}},
$$

where $A$ is a nonzero constant and $\beta_{i} \geq(k+2) /(n-1)(i=$ $1,2, \ldots, t)$.

For the sake of simplicity, we denote

$$
\beta_{1}+\beta_{2}+\cdots+\beta_{t}=q .
$$

From (17), we have

$$
f^{(k)}=\frac{g(z)}{\left(z-z_{1}\right)^{\beta_{1}+k}\left(z-z_{2}\right)^{\beta_{2}+k} \cdots\left(z-z_{t}\right)^{\beta_{t}+k}},
$$

where $g(z)$ is a polynomial such that $\operatorname{deg}(g(z)) \leq k(t-1)$.
From (17) and (19), we get

$$
\begin{aligned}
f^{(k)}- & a f^{n} \\
= & \frac{g(z)}{\left(z-z_{1}\right)^{\beta_{1}+k}\left(z-z_{2}\right)^{\beta_{2}+k} \cdots\left(z-z_{t}\right)^{\beta_{t}+k}} \\
& -\frac{a A^{n}}{\left(z-z_{1}\right)^{n \beta_{1}}\left(z-z_{2}\right)^{n \beta_{2}} \cdots\left(z-z_{t}\right)^{n \beta_{t}}} \\
= & {\left[g(z)\left(z-z_{1}\right)^{(n-1) \beta_{1}-k}\left(z-z_{2}\right)^{(n-1) \beta_{2}-k}\right.} \\
& \left.\cdots\left(z-z_{t}\right)^{(n-1) \beta_{t}-k}-a A^{n}\right] \\
& \times\left(\left(z-z_{1}\right)^{n \beta_{1}}\left(z-z_{2}\right)^{n \beta_{2}} \cdots\left(z-z_{t}\right)^{n \beta_{t}}\right)^{-1} .
\end{aligned}
$$

By the assumption that $f^{(k)}-a f^{n}$ has exactly one zero $z_{0}$ with multiply $l$, we have

$$
f^{(k)}-a f^{n}=\frac{C\left(z-z_{0}\right)^{l}}{\left(z-z_{1}\right)^{n \beta_{1}}\left(z-z_{2}\right)^{n \beta_{2}} \cdots\left(z-z_{t}\right)^{n \beta_{t}}},
$$

where $C$ is a nonzero constant. Thus,

$C\left(z-z_{0}\right)^{l} \equiv g(z)\left(z-z_{1}\right)^{(n-1) \beta_{1}-k} \cdots\left(z-z_{t}\right)^{(n-1) \beta_{t}-k}-a A^{n}$.

Differentiating (22), we obtain

$$
\begin{aligned}
C l\left(z-z_{0}\right)^{l-1} & \\
\equiv & \left(z-z_{1}\right)^{(n-1) \beta_{1}-k-1} \cdots\left(z-z_{t}\right)^{(n-1) \beta_{t}-k-1} \\
\times & {\left[g^{\prime}(z)\left(z-z_{1}\right) \cdots\left(z-z_{t}\right)\right.} \\
& \left.\quad+g(z) \sum_{i=1}^{t}\left((n-1) \beta_{i}-k\right) \prod_{j=1, j \neq i}^{t}\left(z-z_{j}\right)\right] .
\end{aligned}
$$

For the sake of simplicity, we denote

$$
\begin{gathered}
g_{1}(z)=C l\left(z-z_{0}\right)^{l-1}, \\
g_{2}(z)=\left(z-z_{1}\right)^{(n-1) \beta_{1}-k-1} \cdots\left(z-z_{t}\right)^{(n-1) \beta_{t}-k-1} \\
\times\left[g^{\prime}(z)\left(z-z_{1}\right) \cdots\left(z-z_{t}\right)\right. \\
\left.+g(z) \sum_{i=1}^{t}\left((n-1) \beta_{i}-k\right) \prod_{j=1, j \neq i}^{t}\left(z-z_{j}\right)\right] .
\end{gathered}
$$

Hence,

$$
g_{1}(z) \equiv g_{2}(z)
$$


Since $(n-1) \beta_{i}-k-1 \geq 1$, we have $g_{2}\left(z_{i}\right)=0$. But $g_{1}\left(z_{i}\right) \neq 0(i=1,2, \ldots, t)$, a contradiction.

Case 2. If $f^{(k)}-a f^{n}$ has no zeros, then $l=0$ for (21). We have

$$
f^{(k)}-a f^{n}=\frac{C}{\left(z-z_{1}\right)^{n \beta_{1}}\left(z-z_{2}\right)^{n \beta_{2}} \cdots\left(z-z_{t}\right)^{n \beta_{t}}},
$$

where $C$ is a nonzero constant. Thus,

$$
\begin{aligned}
& C \equiv g(z)\left(z-z_{1}\right)^{(n-1) \beta_{1}-k} \cdots\left(z-z_{t}\right)^{(n-1) \beta_{t}-k}-a A^{n}, \\
& g(z)\left(z-z_{1}\right)^{(n-1) \beta_{1}-k} \cdots\left(z-z_{t}\right)^{(n-1) \beta_{t}-k} \equiv C+a A^{n} .
\end{aligned}
$$

Obviously, $g(z)\left(z-z_{1}\right)^{(n-1) \beta_{1}-k} \cdots\left(z-z_{t}\right)^{(n-1) \beta_{t}-k}$ is not a constant, a contradiction.

Lemma 4 is proved.

Lemma 5. Let $f(z)$ be a rational function and $a \in \mathbb{C} \backslash\{0\}$, and $k, n, m \in \mathbb{N}^{+}$with $n \geq 2$ and $m \geq(k+2) /(n-1)$. If all zeros of $f$ are of multiplicity at least $k+1$ and all poles of $f$ are of multiplicity at least $m$, then $f^{(k)}-a f^{n}$ has at least two distinct zeros.

Proof. Suppose, to the contrary, that $f^{(k)}-a f^{n}$ has at most one zero.

Case I. When $f$ is a nonconstant polynomial, noting that all zeros of $f$ have multiplicity at least $k+1$, we know that $f^{(k)}-$ $a f^{n}$ must have zeros. We claim that $f$ has exactly one zero. Otherwise, we can get that $f^{(k)}-a f^{n}$ has at least two zeros, which contradicts our assumption.

Set

$$
f(z)=B\left(z-z_{0}\right)^{s},
$$

where $s \geq k+1, B$ is a nonzero constant. Then

$$
\begin{aligned}
f^{(k)}(z) & -a f^{n}(z) \\
= & B\left(z-z_{0}\right)^{s-k} \\
& \times\left[s(s-1) \cdots(s-k+1)-a\left(z-z_{0}\right)^{(n-1) s+k}\right] .
\end{aligned}
$$

Since $s-k \geq 1$, we obtain that $s(s-1) \cdots(s-k+$ 1) $-a\left(z-z_{0}\right)^{s+k}$ has at least one zero which is not $z_{0}$ from (29). Therefore, $f^{(k)}-a f^{n}$ has at least two distinct zeros, a contradiction.

Case II. When $f$ is rational but not a polynomial, we consider two cases.

Case 1. Suppose that $f^{(k)}-a f^{n}$ has only zero $z_{0}$ with multiplicity at least $l$. If $f \neq 0$, by Lemma 4 , we get a contradiction. So $f$ has zeros, and then we can deduce that $z_{0}$ is the only zero of $f$. Otherwise, $f^{(k)}-a f^{n}$ has at least two distinct zeros, a contradiction.

We set

$$
f(z)=\frac{A\left(z-z_{0}\right)^{s}}{\left(z-z_{1}\right)^{\beta_{1}}\left(z-z_{2}\right)^{\beta_{2}} \cdots\left(z-z_{t}\right)^{\beta_{t}}},
$$

where $A$ is a nonzero constant and $s \geq k+1, \beta_{i} \geq m \geq$ $((k+2) /(n-1))(i=1,2, \ldots, t)$.

For the sake of simplicity, we denote

$$
\beta_{1}+\beta_{2}+\cdots+\beta_{t}=q
$$

From (31), we have

$$
f^{(k)}=\frac{A\left(z-z_{0}\right)^{s-k} g(z)}{\left(z-z_{1}\right)^{\beta_{1}+k}\left(z-z_{2}\right)^{\beta_{2}+k} \cdots\left(z-z_{t}\right)^{\beta_{t}+k}},
$$

where $g(z)$ is a polynomial with $\operatorname{deg}(g) \leq k(s+t+1)$.

From (30) and (32), we get

$$
\begin{aligned}
& f^{(k)}-a f^{n} \\
&= \frac{A\left(z-z_{0}\right)^{s-k} g(z)}{\left(z-z_{1}\right)^{\beta_{1}+k}\left(z-z_{2}\right)^{\beta_{2}+k} \cdots\left(z-z_{t}\right)^{\beta_{t}+k}} \\
&-\frac{A^{n}\left(z-z_{0}\right)^{n s}}{\left(z-z_{1}\right)^{n \beta_{1}}\left(z-z_{2}\right)^{n \beta_{2}} \cdots\left(z-z_{t}\right)^{n \beta_{t}}} \\
&=\left(A\left(z-z_{0}\right)^{s-k}\right. \\
& \times\left[g(z)\left(z-z_{1}\right)^{(n-1) \beta_{1}-k}\left(z-z_{2}\right)^{(n-1) \beta_{2}-k}\right. \\
& \cdots\left(z-z_{t}\right)^{(n-1) \beta_{t}-k}-a A^{n-1} \\
&\left.\left.\quad \times\left(z-z_{0}\right)^{(n-1) s+k}\right]\right) \\
& \quad \times\left(\left(z-z_{1}\right)^{n \beta_{1}}\left(z-z_{2}\right)^{n \beta_{2}} \cdots\left(z-z_{t}\right)^{n \beta_{t}}\right)^{-1} .
\end{aligned}
$$

By assumption that $f^{(k)}-a f^{n}$ has exactly one zero $z_{0}$ with multiplicity $l$, we have

$$
f^{(k)}-a f^{n}=\frac{B\left(z-z_{0}\right)^{l}}{\left(z-z_{1}\right)^{n \beta_{1}}\left(z-z_{2}\right)^{n \beta_{2}} \cdots\left(z-z_{t}\right)^{n \beta_{t}}},
$$

where $B$ is a nonzero constant. Thus,

$$
\begin{aligned}
B\left(z-z_{0}\right)^{l} \equiv & A\left(z-z_{0}\right)^{s-k} \\
\times[ & g(z)\left(z-z_{1}\right)^{(n-1) \beta_{1}-k} \cdots\left(z-z_{t}\right)^{(n-1) \beta_{t}-k} \\
& \left.\quad-a A^{n-1}\left(z-z_{0}\right)^{(n-1) s+k}\right] .
\end{aligned}
$$

Case 1.1. If $l>s-k$, from (35), we can deduce that $z_{0}$ is a zero of $\left(z-z_{1}\right)^{(n-1) \beta_{1}-k} \cdots\left(z-z_{t}\right)^{(n-1) \beta_{t}-k}$, a contradiction.

Case 1.2. If $l=s-k$, from (35), it follows that

$$
\begin{gathered}
g(z)\left(z-z_{1}\right)^{(n-1) \beta_{1}-k} \cdots\left(z-z_{t}\right)^{(n-1) \beta_{t}-k} \\
-a A^{n-1}\left(z-z_{0}\right)^{(n-1) s+k} \equiv \frac{B}{A} .
\end{gathered}
$$


Differentiating (36), we have

$$
\begin{aligned}
& \left(z-z_{1}\right)^{(n-1) \beta_{1}-k-1} \cdots\left(z-z_{t}\right)^{(n-1) \beta_{t}-k-1} \\
& \times\left[g^{\prime}(z)\left(z-z_{1}\right) \cdots\left(z-z_{t}\right)\right. \\
& \left.\quad+g(z) \sum_{i=1}^{t}\left((n-1) \beta_{i}-k\right) \prod_{j=1, j \neq i}^{t}\left(z-z_{j}\right)\right] \\
& \equiv a((n-1) s+k) A^{n-1}\left(z-z_{0}\right)^{(n-1) s+k-1} .
\end{aligned}
$$

For the sake of simplicity, we denote

$$
\begin{aligned}
& g_{1}(z)=\left(z-z_{1}\right)^{(n-1) \beta_{1}-k-1} \cdots\left(z-z_{t}\right)^{(n-1) \beta_{t}-k-1} \\
& \times\left[g^{\prime}(z)\left(z-z_{1}\right) \cdots\left(z-z_{t}\right)\right. \\
& \left.\quad+g(z) \sum_{i=1}^{t}\left((n-1) \beta_{i}-k\right) \prod_{j=1, j \neq i}^{t}\left(z-z_{j}\right)\right], \\
& g_{2}(z)=a((n-1) s+k) A^{n-1}\left(z-z_{0}\right)^{(n-1) s+k-1} .
\end{aligned}
$$

Thus,

$$
g_{1}(z) \equiv g_{2}(z)
$$

Since $(n-1) \beta_{i}-k-1 \geq 1$, we get $g_{1}\left(z_{i}\right)=0$, but $g_{2}\left(z_{i}\right) \neq 0(i=1,2, \ldots, t)$, a contradiction.

Case 2. If $f^{(k)}-a f^{n}$ has no zeros, then $l=0$ for (34). Similarly, as the proof of Case 1, we also have a contradiction.

The proof is completed.

\section{Proofs of Theorems}

Proof. In Theorem 11, suppose that $\mathscr{F}$ is not normal in $D$. Then there exists at least one point $z_{0}$ such that $\mathscr{F}$ is not normal at the point $z_{0}$. Without loss of generality, we assume that $z_{0}=0$. By Lemma 1 , there exist points $z_{j} \rightarrow 0$, positive numbers $\rho_{j} \rightarrow 0$, and functions $f_{j} \in \mathscr{F}$ such that

$$
g_{j}(\xi)=\rho_{j}^{k /(n-1)} f_{j}\left(z_{j}+\rho_{j} \xi\right) \Longrightarrow g(\xi)
$$

locally uniformly with respect to the spherical metric, where $g$ is a nonconstant meromorphic function in $\mathbb{C}$ and whose poles and zeros are of multiplicity at least $d$ and $k$, respectively. Moreover, the order of $g$ is at most 2 .

From (40), we know that

$$
\begin{aligned}
g_{j}^{(k)} & (\xi)=\rho_{j}^{n k /(n-1)} f_{j}^{(k)}\left(z_{j}+\rho_{j} \xi\right) \Longrightarrow g^{(k)}(\xi) \\
g_{j}^{(k)}(\xi) & -a g_{j}^{n}(\xi)-\rho_{j}^{n k /(n-1)} b \\
= & \rho_{j}^{n k /(n-1)}\left(f_{j}^{(k)}\left(z_{j}+\rho_{j} \xi\right)-a f_{j}^{n}\left(z_{j}+\rho_{j} \xi\right)-b\right) \\
\Longrightarrow & g^{(k)}(\xi)-a g^{n}(\xi)
\end{aligned}
$$

also locally uniformly with respect to the spherical metric.
Case 1. If $k=1$, by Theorems 4 and 7 , the Theorem 11 assumes.

Case 2. If $k=2, n \geq 4$, by Theorems 9 and 10 , the Theorem 11 assumes.

Case 3. If $k=2, n=3$ or $k \geq 3,3 \leq n \leq k+1$ (If $n \geq k+2$, by Theorems 9 and 10, we can get Theorem 11).

If $g^{(k)}(\xi)-a g^{n}(\xi) \equiv 0$, since all poles of $g$ have multiplicity at least $d$, we have

$$
\begin{aligned}
n T(r, g) & =T\left(r, g^{n}\right)=T\left(r, g^{(k)}\right)+O(1) \\
& =m\left(r, g^{(k)}\right)+N\left(r, g^{(k)}\right)+O(1) \\
& \leq m(r, g)+N(r, g)+k \bar{N}(r, g)+S(r, g) \\
& \leq T(r, g)+\frac{k(n-2)}{k+1} T(r, g)+S(r, g) \\
& \leq(n-1) T(r, g)+S(r, g) .
\end{aligned}
$$

Therefore, $g(\xi)$ is a constant, a contradiction. So $g^{(k)}(\xi)-$ $\operatorname{ag}^{n}(\xi) \not \equiv 0$.

By Lemma 2, we have

$$
\begin{aligned}
T(r, g) & \leq \frac{1}{k} N\left(r, \frac{1}{g}\right)+\bar{N}\left(r, \frac{1}{g^{(k)}-a g^{n}}\right)+S(r, g) \\
& \leq \frac{1}{k} T\left(r, \frac{1}{g}\right)+\bar{N}\left(r, \frac{1}{g^{(k)}-a g^{n}}\right)+S(r, g) .
\end{aligned}
$$

Then

$$
\begin{gathered}
\left(1-\frac{1}{k}\right) T(r, g) \leq \bar{N}\left(r, \frac{1}{g^{(k)}-a g^{n}}\right)+S(r, g), \\
T(r, g) \leq\left(1+\frac{1}{k-1}\right) \bar{N}\left(r, \frac{1}{g^{(k)}-a g^{n}}\right)+S(r, g) .
\end{gathered}
$$

If $g^{(k)}(\xi)-a g^{n}(\xi) \neq 0$, then (46) gives that $g(\xi)$ is also a constant. Hence, $g^{(k)}(\xi)-a g^{n}(\xi)$ is a nonconstant meromorphic function and has at least one zero.

Next we prove that $g^{(k)}(\xi)-a g^{n}(\xi)$ has just a unique zero. On the contrary, let $\xi_{0}$ and $\xi_{0}^{*}$ be two distinct zeros of $g^{(k)}(\xi)-$ $a g^{n}(\xi)$, and choose $\delta(>0)$ small enough such that $D\left(\xi_{0}, \delta\right) \cap$ $D\left(\xi_{0}^{*}, \delta\right)=\phi$, where $D\left(\xi_{0}, \delta\right)=\left\{\xi:\left|\xi-\xi_{0}\right|<\delta\right\}$ and $D\left(\xi_{0}^{*}, \delta\right)=$ $\left\{\xi:\left|\xi-\xi_{0}^{*}\right|<\delta\right\}$. From (42), by Hurwitz's theorem, there exist points $\xi_{j} \in D\left(\xi_{0}, \delta\right), \xi_{j}^{*} \in D\left(\xi_{0}^{*}, \delta\right)$ such that for sufficiently large $j$

$$
\begin{aligned}
& f_{j}^{(k)}\left(z_{j}+\rho_{j} \xi_{j}\right)-a f_{j}^{n}\left(z_{j}+\rho_{j} \xi_{j}\right)-b=0, \\
& f_{j}^{(k)}\left(z_{j}+\rho_{j} \xi_{j}^{*}\right)-a f_{j}^{n}\left(z_{j}+\rho_{j} \xi_{j}^{*}\right)-b=0 .
\end{aligned}
$$

By the hypothesis that for each pair of functions $f$ and $g$ in $\mathscr{F}, f^{(k)}-a f^{n}$ and $g^{(k)}-a g^{n}$ share $b$ in $D$, we know that for any positive integer $m$

$$
\begin{aligned}
& f_{m}^{(k)}\left(z_{j}+\rho_{j} \xi_{j}\right)-a f_{m}^{n}\left(z_{j}+\rho_{j} \xi_{j}\right)-b=0, \\
& f_{m}^{(k)}\left(z_{j}+\rho_{j} \xi_{j}^{*}\right)-a f_{m}^{n}\left(z_{j}+\rho_{j} \xi_{j}^{*}\right)-b=0 .
\end{aligned}
$$


Fix $m$, take $j \rightarrow \infty$, and note $z_{j}+\rho_{j} \xi_{j} \rightarrow 0, z_{j}+\rho_{j} \xi_{j}^{*} \rightarrow$ 0 ; then $f_{m}^{(k)}(0)-a f_{m}^{n}(0)-b=0$. Since the zeros of $f_{m}^{(k)}-a f_{m}^{n}-b$ have no accumulation point, so

$$
z_{j}+\rho_{j} \xi_{j}=0, \quad z_{j}+\rho_{j} \xi_{j}^{*}=0 .
$$

Hence, $\xi_{j}=-z_{j} / \rho_{j}, \xi_{j}^{*}=-z_{j} / \rho_{j}$. This contradicts $\xi_{j} \in$ $D\left(\xi_{0}, \delta\right), \xi_{j}^{*} \in D\left(\xi_{0}^{*}, \delta\right)$ and $D\left(\xi_{0}, \delta\right) \cap D\left(\xi_{0}^{*}, \delta\right)=\phi$. So $g^{(k)}(\xi)-a g^{n}(\xi)$ has just a unique zero, which can be denoted by $\xi_{0}$.

Noting that $g$ has poles and zeros of multiplicities at least $d$ and $k$, respectively, (46) deduces that $g(\xi)$ is a rational function with degree at most 2 .

If $g(\xi)$ is a polynomial, noting that $\operatorname{deg} g \leq 2$ and the multiplicities of zeros are at least $k$, we have $k=2$ and $n=3$. Hence, there exist $\xi_{1}$ and $c \neq 0$ such that $g(\xi)=c\left(\xi-\xi_{1}\right)^{2}$, and then $g^{\prime \prime}(\xi)-a g^{3}(\xi)=c\left(2-a c^{2}\left(\xi-\xi_{1}\right)^{6}\right)$ has 6 distinct zeros, a contradiction.

Suppose that $g(\xi)$ is not a polynomial, $k=2$ and $n=3$. Then the multiplicities of poles of $g(\xi)$ are at least $(k+1) /(n-$ $2)=3$, which implies that $\operatorname{deg} g \geq 3$, a contradiction.

Suppose that $g(\xi)$ is not a polynomial, $k \geq 3$ and $3 \leq n \leq$ $k+1$; we distinguish two cases.

Case $i$. If $g(\xi)$ has zeros, since all zeros of $g(\xi)$ have multiplicity at least $k(\geq 3)$, it follows that $\operatorname{deg} g \geq 3$, a contradiction.

Case ii. If $g(\xi) \neq 0$, then (46) should be as follows

$$
T(r, g) \leq \bar{N}\left(r, \frac{1}{g^{(k)}-a g^{n}}\right)+S(r, g) .
$$

From (50), we can see that $g(\xi)$ is a rational function with degree at most 1 . Since all poles of $g(\xi)$ have multiplicity at least $d(\geq(k+1) /(n-2) \geq(k+1) /(k-1)>1)$, which gives that $\operatorname{deg} g \geq 2$, a contradiction.

This completes the proof of Theorem 11.

Proof. In Theorem 13, suppose that $\mathscr{F}$ is not normal in $D$. Then there exists at least one point $z_{0}$ such that $\mathscr{F}$ is not normal at the point $z_{0}$. Without loss of generality, we assume that $z_{0}=0$. By Lemma 1 , there exist points $z_{j} \rightarrow 0$, positive numbers $\rho_{j} \rightarrow 0$, and functions $f_{j} \in \mathscr{F}$ such that

$$
g_{j}(\xi)=\rho_{j}^{k} f_{j}\left(z_{j}+\rho_{j} \xi\right) \Longrightarrow g(\xi)
$$

locally uniformly with respect to the spherical metric, where $g$ is a nonconstant meromorphic function in $\mathbb{C}$ and whose poles and zeros are of multiplicity at least $k+2$ and $k+1$, respectively. Moreover, the order of $g$ is at most 2 .

From (51), we know

$$
\begin{aligned}
& g_{j}^{(k)}(\xi)=\rho_{j}^{2 k} f_{j}^{(k)}\left(z_{j}+\rho_{j} \xi\right) \Longrightarrow g^{(k)}(\xi), \\
& g_{j}^{(k)}(\xi)-a g_{j}^{2}(\xi)-\rho_{j}^{2 k} b \\
&=\rho_{j}^{2 k}\left(f_{j}^{(k)}\left(z_{j}+\rho_{j} \xi\right)-a f_{j}^{2}\left(z_{j}+\rho_{j} \xi\right)-b\right) \\
& \Longrightarrow g^{(k)}(\xi)-a g^{2}(\xi)
\end{aligned}
$$

also locally uniformly with respect to the spherical metric.
If $g^{(k)}(\xi)-a g^{2}(\xi) \equiv 0$, since all poles of $f$ have multiplicity at least $k+2$, we can deduce that $g(\xi)$ is an entire function easily. Thus,

$$
\begin{aligned}
2 T(r, g) & =T\left(r, g^{2}\right)=T\left(r, g^{(k)}\right)+O(1) \\
& =m\left(r, g^{(k)}\right)+N\left(r, g^{(k)}\right)+O(1) \\
& \leq m(r, g)+N(r, g)+k \bar{N}(r, g)+S(r, g) \\
& \leq T(r, g)+S(r, g) .
\end{aligned}
$$

Therefore, $g(\xi)$ is a constant, a contradiction. So $g^{(k)}(\xi)-$ $a g^{2}(\xi) \quad \equiv \quad 0$. By Lemmas 3, 4, and 5, $g^{(k)}(\xi)-a g^{2}(\xi)$ has at least two distinct zeros. Proceeding as in the later proof of Theorem 11, we will get a contradiction. The proof is completed.

Similarly, as the proof of Theorem 11, when $f$ is a holomorphic function, we can get the following theorem which has been gotten in [23].

Theorem 18 (see [23]). Let $D$ be a domain in $\mathbb{C}$ and let $\mathscr{F}$ be a family of holomorphic functions in D. Let $k, n \in \mathbb{N}^{+}, n \geq 2$ and let $a, b$ be two finite complex numbers with $a \neq 0$. Suppose that every $f \in \mathscr{F}$ has all its zeros of multiplicity at least $k$. If $f^{(k)}-a f^{n}$ and $g^{(k)}-a g^{n}$ share the value $b$ IM for every pair of functions $(f, g)$ of $\mathscr{F}$, then $\mathscr{F}$ is a normal family in $D$.

\section{Acknowledgments}

This paper is supported by the Nature Science Foundation of China (11271090), Nature Science Foundation of Guangdong Province (S2012010010121), and Graduate Research and Innovation Projects of Xinjiang Province (XJGRI2013131). This work was supported by the Visiting Scholar Program of Chern Institute of Mathematics at Nankai University. The second author would like to express his hearty gratitude to Chern Institute of Mathematics which provided very comfortable research environments to him where he worked as a Visiting Scholar.

\section{References}

[1] C. C. Yang and H. X. Yi, Uniqueness Theory of Meromorphic Functions, vol. 557 of Mathematics and Its Applications, Science Press, Beijing, China; Kluwer Academic Publishers, New York, NY, USA, 2003.

[2] Y. X. Gu, X. C. Pang, and M. L. Fang, Theory of Normal Family and Its Applications, Science Press, Beijing, China, 2007 (Chinese).

[3] W. K. Hayman, Meromorphic Functions, Oxford Mathematical Monographs, Clarendon Press, Oxford, UK, 1964.

[4] L. Yang, Value Distribution Theory, Springer, Berlin, Germany, 1993.

[5] W. Schwick, "Normality criteria for families of meromorphic functions," Journal d'Analyse Mathématique, vol. 52, pp. 241289, 1989. 
[6] X. C. Pang and L. Zalcman, "Normal families and shared values," The Bulletin of the London Mathematical Society, vol. 32, no. 3, pp. 325-331, 2000.

[7] X. C. Pang and L. Zalcman, "Normality and shared values," Arkiv för Matematik, vol. 38, no. 1, pp. 171-182, 2000.

[8] Q. C. Zhang, "Normal families of meromorphic functions concerning shared values," Journal of Mathematical Analysis and Applications, vol. 338, no. 1, pp. 545-551, 2008.

[9] W. K. Hayman, Research Problems in Function Theory, The Athlone Press University of London, London, UK, 1967.

[10] D. Drasin, "Normal families and the Nevanlinna theory," Acta Mathematica, vol. 122, pp. 231-263, 1969.

[11] X. C. Pang, "On normal criterion of meromorphic functions," Science in China A, vol. 33, no. 5, pp. 521-527, 1990.

[12] H. H. Chen and M. L. Fang, "On a theorem of Drasin," Advances in Mathematics, vol. 20, pp. 504-504, 1991 (Chinese).

[13] Y. S. Ye, "A new normality criterion and its application," Chinese Annals of Mathematics A, vol. 12, supplement, pp. 44-49, 1991.

[14] H. H. Chen and Y. X. Gu, "An improvement of Marty's criterion and its application," Science in China A, vol. 36, no. 6, pp. 674681, 1993.

[15] S. Y. Li, "On normality criterion of a class of the functions," Journal of Fujian Normal University, vol. 2, pp. 156-158, 1984.

[16] X. J. Li, "Proof of Hayman's conjecture on normal families," Science in China A, vol. 28, no. 6, pp. 596-603, 1985.

[17] J. K. Langley, "On normal families and a result of Drasin," Proceedings of the Royal Society of Edinburgh A, vol. 98, no. 3-4, pp. 385-393, 1984.

[18] L. Zalcman, On Some Questions of Hayman, 1994.

[19] M. L. Fang and W. J. Yuan, "On the normality for families of meromorphic functions," Indian Journal of Mathematics, vol. 43, no. 3, pp. 341-351, 2001.

[20] W. J. Yuan, J. J. Wei, and J. M. Lin, "A note on normal families of meromorphic functions concerning shared values," Discrete Dynamics in Nature and Society, vol. 2011, Article ID 463287, 10 pages, 2011.

[21] J. H. Wang, Q. Lu, and Q. L. Liao, "Normal criteria and shared values by differential polynomials," Advances in Pure Mathematics, vol. 1, no. 4, pp. 210-217, 2011.

[22] W. J. Yuan, Z. R. Li, and J. M. Lin, "Some normality criteria of function families concerning shared values," Mathematical Methods in the Applied Sciences, vol. 35, no. 17, pp. 2095-2100, 2012.

[23] H. L. Qiu, D. Liu, and M. L. Fang, "Hayman's question concerning normal families and shared values," Scientia Sinica Mathematica, vol. 42, no. 6, pp. 603-610, 2012 (Chinese).

[24] L. Zalcman, "A heuristic principle in complex function theory," The American Mathematical Monthly, vol. 82, no. 8, pp. 813-817, 1975.

[25] L. Zalcman, "Normal families: new perspectives," Bulletin of the American Mathematical Society, vol. 35, no. 3, pp. 215-230, 1998. 


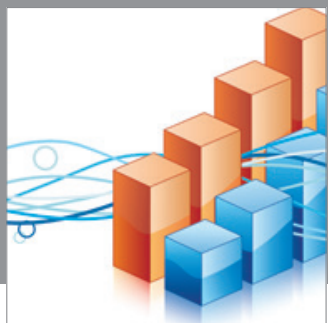

Advances in

Operations Research

mansans

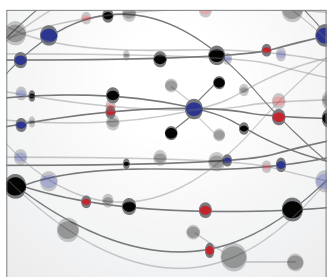

The Scientific World Journal
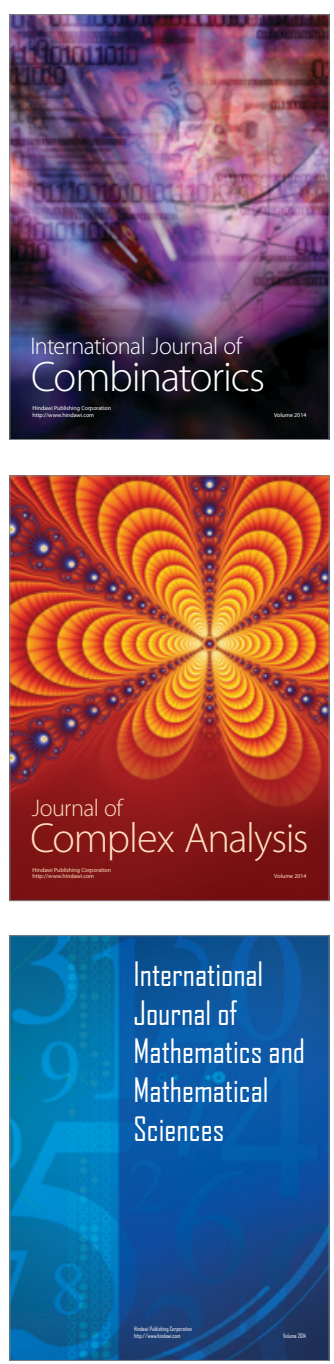
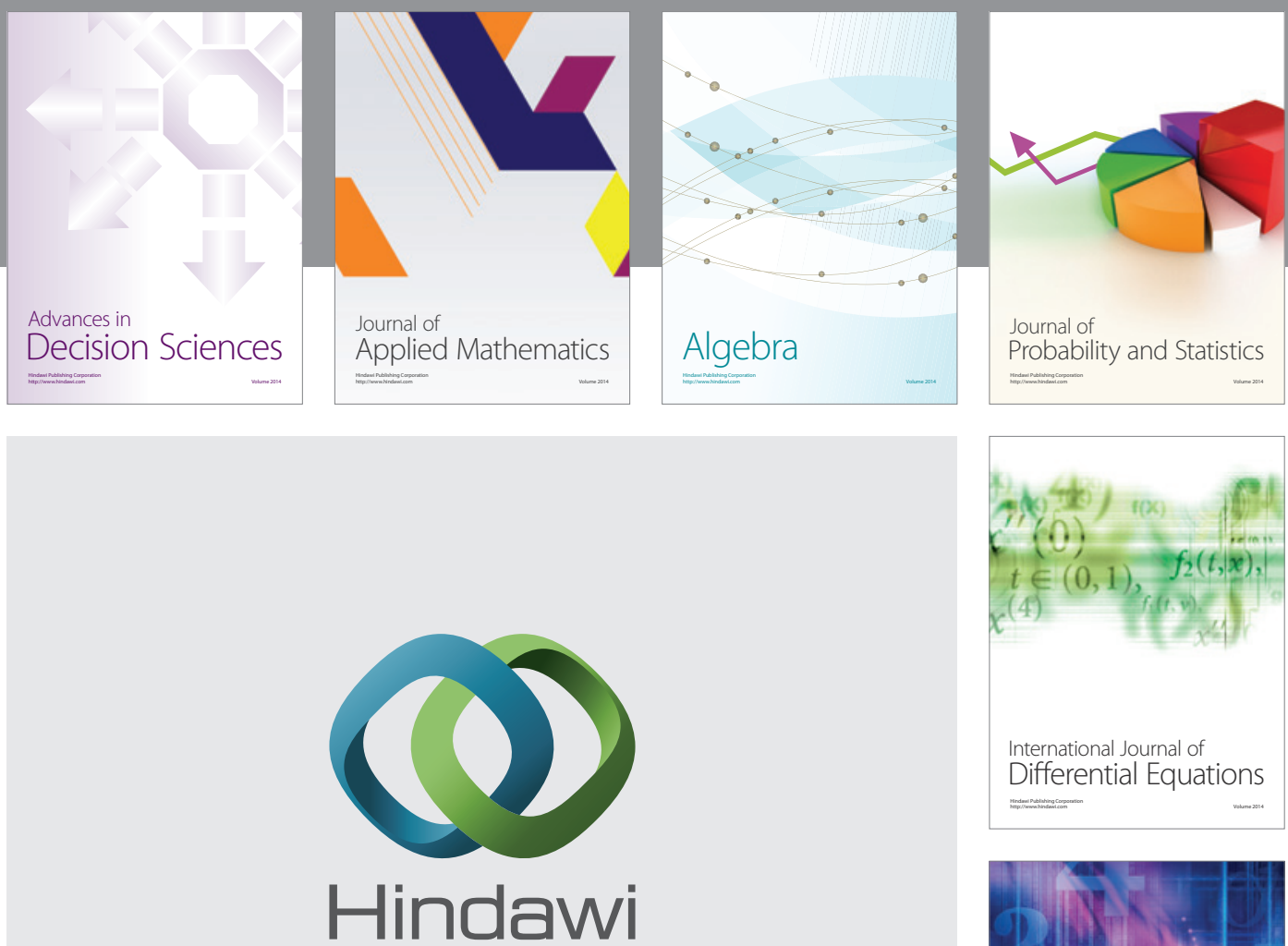

Submit your manuscripts at http://www.hindawi.com
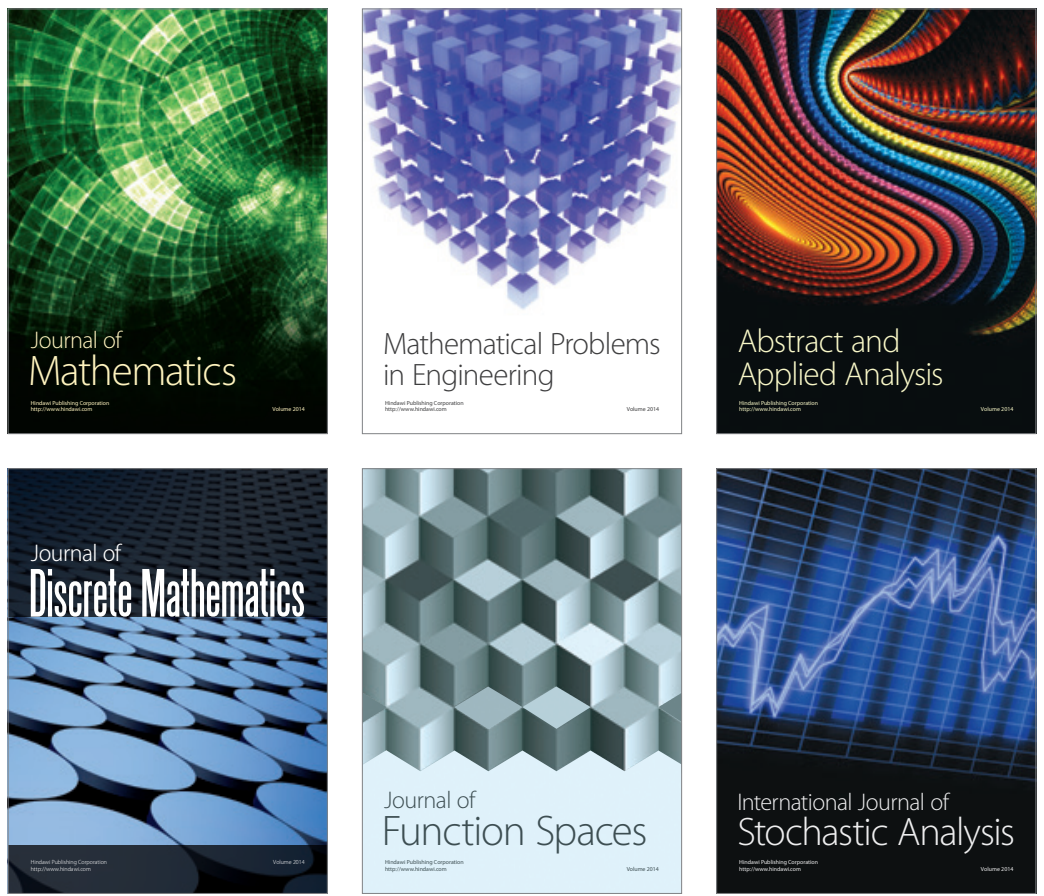

Journal of

Function Spaces

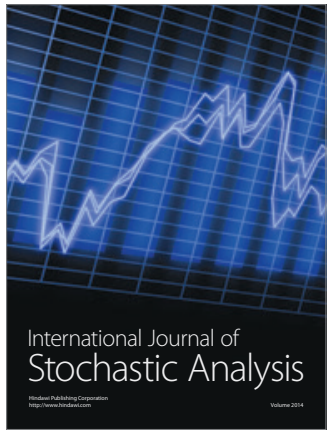

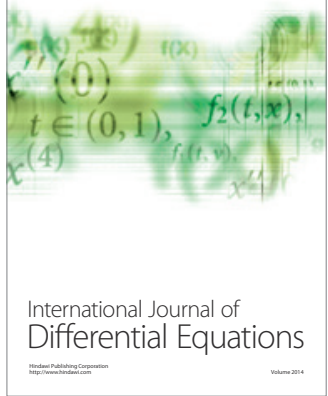
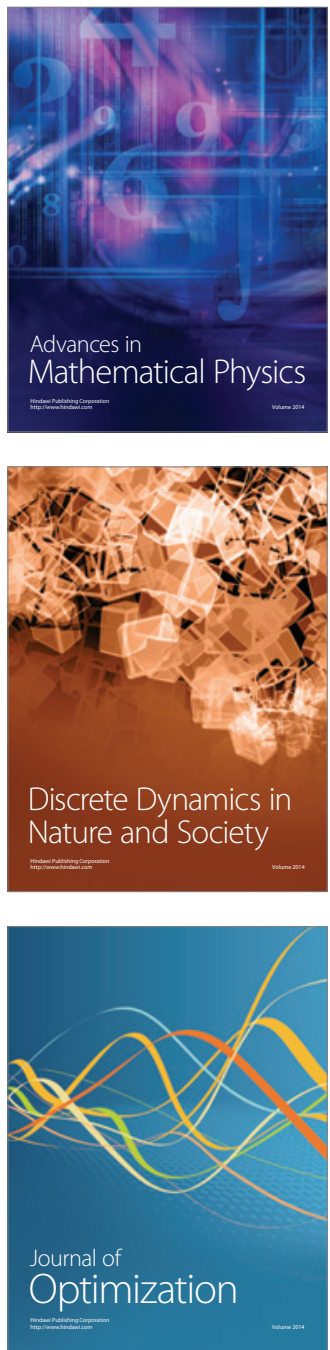